\title{
Functional test of PCDHB11, the most human-specific neuronal surface protein
}

\author{
Guilherme Braga de Freitas, Rafaella Araújo Gonçalves and Matthias Gralle*
}

\begin{abstract}
Background: Brain-expressed proteins that have undergone functional change during human evolution may contribute to human cognitive capacities, and may also leave us vulnerable to specifically human diseases, such as schizophrenia, autism or Alzheimer's disease. In order to search systematically for those proteins that have changed the most during human evolution and that might contribute to brain function and pathology, all proteins with orthologs in chimpanzee, orangutan and rhesus macaque and annotated as being expressed on the surface of cells in the human central nervous system were ordered by the number of human-specific amino acid differences that are fixed in modern populations.

Results: PCDHB11, a beta-protocadherin homologous to murine cell adhesion proteins, stood out with 12 substitutions and maintained its lead after normalizing for protein size and applying weights for amino acid exchange probabilities. Human PCDHB11 was found to cause homophilic cell adhesion, but at lower levels than shown for other clustered protocadherins. Homophilic adhesion caused by a PCDHB11 with reversion of humanspecific changes was as low as for modern human PCDHB11; while neither human nor reverted PCDHB11 adhered to controls, they did adhere to each other. A loss of function in PCDHB11 is unlikely because intra-human variability did not increase relative to the other human beta-protocadherins.

Conclusions: The brain-expressed protein with the highest number of human-specific substitutions is PCDHB11. In spite of its fast evolution and low intra-human variability, cell-based tests on the only proposed function for PCDHB11 did not indicate a functional change.
\end{abstract}

Keywords: Human evolution, Neutral evolution, Gene ontology, Clustered protocadherins

\section{Background}

Human brains are different from other primate brains. However, it is not clear if the difference is simply a matter of size [1] or if there are any molecular and cellular differences that would help explain uniquely human capabilities. One molecular difference that might be important for the acquisition of such a capability, namely of speech, is the substitution of two amino acids in the protein FOXP2 [2-4]. Other human-specific differences have been discovered in proteins expressed in the brain, but have not yet been linked to a behavioral phenotype [5-8].

It has been surmised for more than 30 years that the main genetic differences between humans and chimpanzees lie in regulatory sequences [9], and recent studies

\footnotetext{
* Correspondence: gralle@bioqmed.ufrj.br

Instituto de Bioquímica Médica Leopoldo de Meis, Universidade Federal do Rio de Janeiro, Rio de Janeiro, Brazil
}

(c) 2016 de Freitas et al. Open Access This article is distributed under the terms of the Creative Commons Attribution 4.0 International License (http://creativecommons.org/licenses/by/4.0/), which permits unrestricted use, distribution, and reproduction in any medium, provided you give appropriate credit to the original author(s) and the source, provide a link to the Creative Commons license, and indicate if changes were made. The Creative Commons Public Domain Dedication waiver (http://creativecommons.org/publicdomain/zero/1.0/) applies to the data made available in this article, unless otherwise stated.

have begun to identify some of the more relevant human-specific elements [10-13]. However, even with the increasing number of sequenced human and other primate genomes, it remains more difficult to identify functionally relevant differences in non-coding parts of the genome, even in well-studied transcription factor binding sites [14], than in protein-coding sequences. Therefore, the present study concentrates on differences in the protein-coding parts of the genome.

While the genomes of individuals from extinct human populations are exciting sources of information on human evolution [15-19], we lack reliable information on the cognitive capabilities of those populations [20, 21]. Therefore, the most useful comparison is with living primates, on whose cognitive phenotype we do have information [22, 23]: a DNA variant might contribute to human cognitive capabilities if it is present in all cognitively normal modern humans, but not in the aligned 
genomes of other primates. The analysis of primate genomes has yielded lists of such variants [24-27]; however, the statistical tests for positive selection are necessarily of low statistical power and selectivity, and the necessary biochemical analysis of such candidate genes has rarely been reported [28].

The initial auto-organization of the human brain and its subsequent forming by the environment are mediated by cell surface proteins [29-31]. Variation in adhesion proteins has been shown to degrade [32] and, possibly, to explain part of normal variation in cognitive function [33]. While the example of FOXP2 shows that intracellular proteins, such as transcription factors, may be important targets of change, the function of proteins present at the cell membrane, such as receptors, channels and adhesion proteins, is more straightforward to quantify. Therefore, the bioinformatical strategy used in the present study was to rank all proteins present on the surface of human central nervous system cells by the number of substitutions they have accumulated on the human lineage. The aim of this strategy was to select a neural cell surface protein with high probability of having changed its function on the human lineage, so that this functional change could be tested for in biological model systems.

\section{Results}

\section{Selection of candidate protein}

A comparison of the protein-coding regions of the reference chimpanzee, orangutan and rhesus macaque genomes to 100 haploid human genomes, sampled from diverse human populations, has been published before [34] and resulted in a list of amino acid positions where all modern human genomes agree with each other and are different from the non-human primates. Almost half of all human proteins contain at least one such amino acid (Fig. 1).

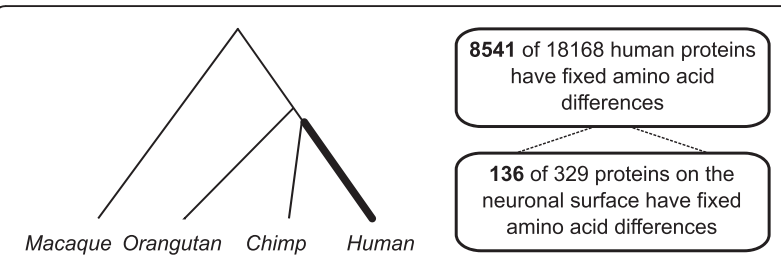

Fig. 1 Pipeline for discovering human-specific amino acid substitutions. Amino acids in human proteins were considered humanspecific wherever they differed from the consensus between the exomes of Macaca mulatta, Pongo abelii and Pan troglodytes. Differences were considered fixed if the human-specific amino acid recurred in 100 haploid human genomes. Among those proteins that could be aligned between the four genomes, the indicated number of proteins contains at least one fixed human-specific difference
The present study focuses on the substitutions occurring in brain cell-surface proteins, i.e. the products of genes annotated both as being expressed in central nervous system cells and as present on the extracellular side of the plasma membrane, according to Gene Ontology [35]. Among 329 proteins in this set, 136 contain at least one fixed human-specific difference (Fig. 1). An unknown fraction of these human-specific substitutions may have had functional consequences. While ideally the functional consequences might be estimated from the position of a substitution within the threedimensional structure of a protein, especially if structure-function relationships are well established, such structural data are not available for many of the candidate proteins. Alternatively, reasoning that a change in function may require several amino acid substitutions or that a change, once it has occurred, may release a functional restraint and permit additional substitutions to occur, the 136 candidate proteins were ordered by the number of fixed human-specific amino acid differences, with $\beta$-protocadherin 11 (PCDHB11) appearing at the top of the list, due to its 12 substitutions (Table 1).

While a high number of substitutions does not necessarily indicate a change in function, several aspects make PCDHB11 stand out from other proteins on the list. Higher numbers of substitutions would be expected to occur by chance in longer proteins; the absolute number was therefore divided by the length of the protein to exclude this explanation. The high rate of substitutions in several proteins, such as PCDH15, can readily be explained by their large size, but PCDHB11 continues to stand out (Table 1). Furthermore, in exome comparisons, some amino acids are frequently found in substitution for each other, probably because their exchange has a lower impact on the function of the protein. The rate of amino acid exchange, when comparing proteins in closely related species, was used in order to weigh the importance of an exchange; amino acids that rarely substitute for each other were given higher weights. When summing up the weights for all the substitutions, PCDHB11 continues to have a score well above those of all the other candidate proteins (Table 1), due to several evolutionarily rare amino acid exchanges, e.g. asparagine to isoleucine and arginine to isoleucine (Table 2).

Functional data show the importance of the distribution of the substitutions among the domains of the protein. Clustered protocadherins are proposed to serve as adhesion proteins that may regulate synaptic contacts between neurons [36, 37]. So far, the function of murine, but not human, clustered protocadherins has been tested in cell culture models and intact organisms [38-50]. In cell culture, among six extracellular cadherin repeats, one transmembrane and one cytoplasmic domain, the 
Table 1 Proteins on the surface of central nervous system cells that have accumulated the highest number of amino acid substitutions on the human lineage

\begin{tabular}{|c|c|c|c|c|c|}
\hline Protein & Substitutions & Length of protein & Subst./length & Weighted subst. & Weighted subst./length \\
\hline PCDHB11 & 12 & 797 & 0.015 & 89 & 0.112 \\
\hline ICAM1 & 6 & 532 & 0.011 & 41 & 0.077 \\
\hline HTR3E & 4 & 456 & 0.009 & 33 & 0.072 \\
\hline $\mathrm{HRH} 1$ & 5 & 487 & 0.010 & 31 & 0.064 \\
\hline DRD5 & 5 & 477 & 0.010 & 30 & 0.063 \\
\hline PCDHB13 & 6 & 798 & 0.008 & 47 & 0.059 \\
\hline GLRA4 & 4 & 417 & 0.010 & 24 & 0.058 \\
\hline HOME3 & 2 & 361 & 0.006 & 19 & 0.053 \\
\hline PCDHB6 & 5 & 794 & 0.006 & 40 & 0.050 \\
\hline VIPR1 & 2 & 457 & 0.004 & 20 & 0.044 \\
\hline CCKAR & 2 & 428 & 0.005 & 18 & 0.042 \\
\hline PCDHB12 & 4 & 797 & 0.005 & 30 & 0.038 \\
\hline OXYR & 3 & 389 & 0.008 & 14 & 0.036 \\
\hline GRIN3A & 6 & 1115 & 0.005 & 38 & 0.034 \\
\hline PCDHB10 & 3 & 797 & 0.004 & 27 & 0.034 \\
\hline SEMA5B & 5 & 1151 & 0.004 & 32 & 0.028 \\
\hline PCDHA5 & 4 & 936 & 0.004 & 26 & 0.028 \\
\hline PCDHB14 & 4 & 798 & 0.005 & 21 & 0.026 \\
\hline GRIN2C & 4 & 1236 & 0.003 & 32 & 0.026 \\
\hline PCDHA2 & 4 & 948 & 0.004 & 23 & 0.024 \\
\hline CD44 & 3 & 742 & 0.004 & 17 & 0.023 \\
\hline PCDHB15 & 4 & 787 & 0.005 & 18 & 0.023 \\
\hline PCDH15 & 9 & 1955 & 0.005 & 43 & 0.022 \\
\hline
\end{tabular}

For each protein, the number of human-specific substitutions, divided by the length of the protein, gives the fraction of amino acids that have changed and become fixed on the human lineage. For each amino acid substitution on the human lineage, a weight was derived from the BLOSUM100 matrix that reflects the rareness of this exchange between organisms that have $99 \%$ amino acid identity, and the weights of all substitutions were summed up for each protein

ones most important for protocadherin specificity are EC2 and EC3 [43], and nine of the changes in human PCDHB11 are concentrated in these two domains (Table 2), suggesting again that they might be relevant.

Very recently, crystal structures of the EC1-3 domains of several murine protocadherins, among them the $\beta$ protocadherin PCDHB1, have been published [51, 52]. By homology to the crystal structure of monomeric PCDHB1 EC1-3 [51], all ten human-specific amino acids in these domains of PCDHB11 are expected to be at least partly exposed to water; such surface-exposed amino acids are less constrained by the structure and may therefore be more variable, unless they contribute to dimer interfaces. In this regard, it is relevant to note that Thr185 in the PCDHB1 structure, corresponding to human-specific PCDHB11 Ser213, hydrogen bonds with Thr143, which was shown to be necessary for protocadherin dimerization in a cell-based assay [51]. Furthermore, the residue corresponding to human-specific PCDHB11 Ser134 contributes to crystal contacts in certain $\gamma$-protocadherins, and so do the EC2 $\beta 4-\beta 5$, the
Phe- $\mathrm{X}_{10}$-Phe loop and the EC3 $\beta 7$ loop, which in PCDHB11 are predicted to contain human-specific Ile185, Phe281 and His336, respectively [52].

While crystal contacts are not evidence of functional importance, and different clustered protocadherins may dimerize slightly differently, the homologies mentioned would suggest, on a purely structural basis, that some of the human-specific mutations might affect the adhesivity of PCDHB11. However, these putative conclusions from bioinformatical investigation depend on functional confirmation.

\section{Functional test of human PCDHB11}

Following an established protocol [43], human PCDHB11 and a well-investigated murine control, PCDHGA3, were electroporated into the normally non-adhesive human suspension cell line K562. Murine PCDHGA3 caused the appearance of large cell clusters, as previously described (Fig. 2c, f); cells expressing human PCDHB11 formed clusters, but smaller ones (Fig. 2b). The proportion of larger cell clusters among all cells was quantified in four 
Table 2 Differences between non-human primate consensus and human PCDHB11

\begin{tabular}{|c|c|c|c|c|}
\hline Position & Consensus & Human & Weight & Domain \\
\hline 4 & Glu & Gln & 3 & Signal peptide \\
\hline 106 & Phe & Leu & 5 & $\mathrm{EC} 1^{\mathrm{a}}$ \\
\hline 134 & Leu & Ser & 11 & $\mathrm{EC} 2^{\mathrm{a}}$ \\
\hline 185 & Asn & Ile & 12 & $E C 2^{a}$ \\
\hline 213 & Thr & Ser & 3 & $\mathrm{EC} 2^{\mathrm{a}}$ \\
\hline 252 & Pro & $\operatorname{Arg}$ & 10 & $E C 3^{\mathrm{a}}$ \\
\hline 263 & Thr & Ile & 8 & $E C 3^{a}$ \\
\hline 281 & Leu & Phe & 5 & $E C 3^{\mathrm{a}}$ \\
\hline 304 & Thr & $\operatorname{Arg}$ & 8 & $\mathrm{EC} 3^{\mathrm{a}}$ \\
\hline 334 & Arg & Ile & 12 & $E C 3^{\mathrm{a}}$ \\
\hline 336 & Gln & His & 4 & $\mathrm{EC} 3^{\mathrm{a}}$ \\
\hline 724 & Arg & Ser & 8 & Cytoplasmic \\
\hline
\end{tabular}

Differences between modern human PCDHB11 and the consensus of Macaca mulatta, Pongo abelii and Pan troglodytes. Human is the consensus of 100 chromosomes, wherever it differs from the ancestral amino acid. Weight is derived from the evolutionary rate of exchange of each amino acid pair (for details see "Methods"). The table shows the amino acids in the constructs used in the experiments; current data show that there is indeed variation within modern humans at the signal peptide site. ${ }^{\mathrm{a} E C 1,} \mathrm{EC2}$, EC3: extracellular cadherin domains $1-3$

independent transfections; the adhesive strength conferred by human PCDHB11 was significant when compared to negative controls, but significantly lower than that conferred by murine PCDHGA3 (Fig. 2g, h).

The weak adhesivity of PCDHB11-transfected cells might be explained by low expression levels. However, the expression of PCDHB11 fused to green fluorescent protein (GFP) was easily detected by Western Blot (Fig. 3a). It has been shown that N-terminal hemagglutinine (HA) tags, which allow selective staining of surfaceexposed proteins, do not reduce the adhesion mediated by $\gamma$-protocadherins [43]. Reasoning that an $\mathrm{N}$-terminal HA-tag would also preserve any adhesion mediated by PCDHB11, cells were transfected with HA-PCDHB11GFP or murine HA-PCDHGA3. HA-PCDHB11-GFP was expressed at much lower levels (Fig. 3b), and this was reflected in lower surface expression (Fig. 3e-g) and a lower proportion of large cell clusters (Fig. 3c, d) than with HA-PCDHGA3. Nevertheless, PCDHB11 was expressed and reached the cell surface.

One reason for the low adhesivity of human PCDHB11 would be a loss of function as consequence of one or more of the mutations that occurred during human evolution. In order to test for an effect of the human-specific substitutions, a protein with reversion of these substitutions to the consensus sequence of nonhuman primate PCDHB11 proteins (Table 2) was synthesized and expressed in $\mathrm{K} 562$ cells. This reverted PCDHB11 had adhesive properties indistinguishable from modern human PCDHB11 (Fig. 2b, e, g, h). While it is not possible to exclude subtle changes, based on the present data, a complete loss or gain of adhesivity, due to the human-specific substitutions, can be refuted.

The properties of both reverted and modern human PCDHB11 were also investigated by quantifying coclustering with other cells. Different populations of K562 cells were transfected with protocadherins and either green or red fluorescent protein, and the two populations were mixed on the following day. As expected, K562 cells without any protocadherin did not form clusters (Fig. 4a), nor did cells transfected with a protocadherin form mixed clusters with negative controls (Fig. 4b-d, e, i, m). Cells transfected with PCDHGA3 and PCDHB11, respectively, did not adhere to each other, which would manifest as a high proportion of mixed clusters (Fig. 4h, l, n, o). However, two cell populations, each transfected with murine PCDHGA3, adhered strongly to each other, as expected (Fig. 4p). In mixtures of modern and ancestral PCDHB11-expressing cells, some mixed clusters were observed (Fig. 4f, g, j, k), which were smaller than the PCDHGA3 clusters. The co-occurrence of green and red cells in the same clusters was quantified in each condition (Fig. 4q). PCDHB11expressing cells adhered more to each other than to negative controls or to murine PCDHGA3. The humanspecific changes did not abolish the weak mutual adhesion.

\section{Intra-human variability in PCDHB11}

Since these assays did not reveal a change in the adhesion function of PCDHB11, the high number of humanspecific amino acid substitutions in PCDHB11 might be thought to be due to a higher local mutational burden or reduced purifying selection. Such processes would necessarily increase variability among humans. However, it has been reported before, in an ethnically homogeneous sample, that human PCDHB11 had lower variability than other $\beta$-protocadherin genes [53]. Here, the numbers of non-synonymous minor alleles in all human $\beta$ protocadherins were reanalyzed using dbSNP; they ranged from 92 to 167 variable positions, and the number for PCDHB11 was 139 , coincidentally the median of the distribution (Fig. 5, open diamonds). This normal intra-human variability of PCDHB11 stands in contrast to the evolutionary data, where PCDHB11 has a much higher number of human-specific amino acid substitutions than any other $\beta$-protocadherin (Table 1; Fig. 5, closed circles).

\section{Discussion}

The aim of this work was to search systematically for the brain protein that has changed the most during human evolution and that might contribute to uniquely human brain function and pathology [23]. 


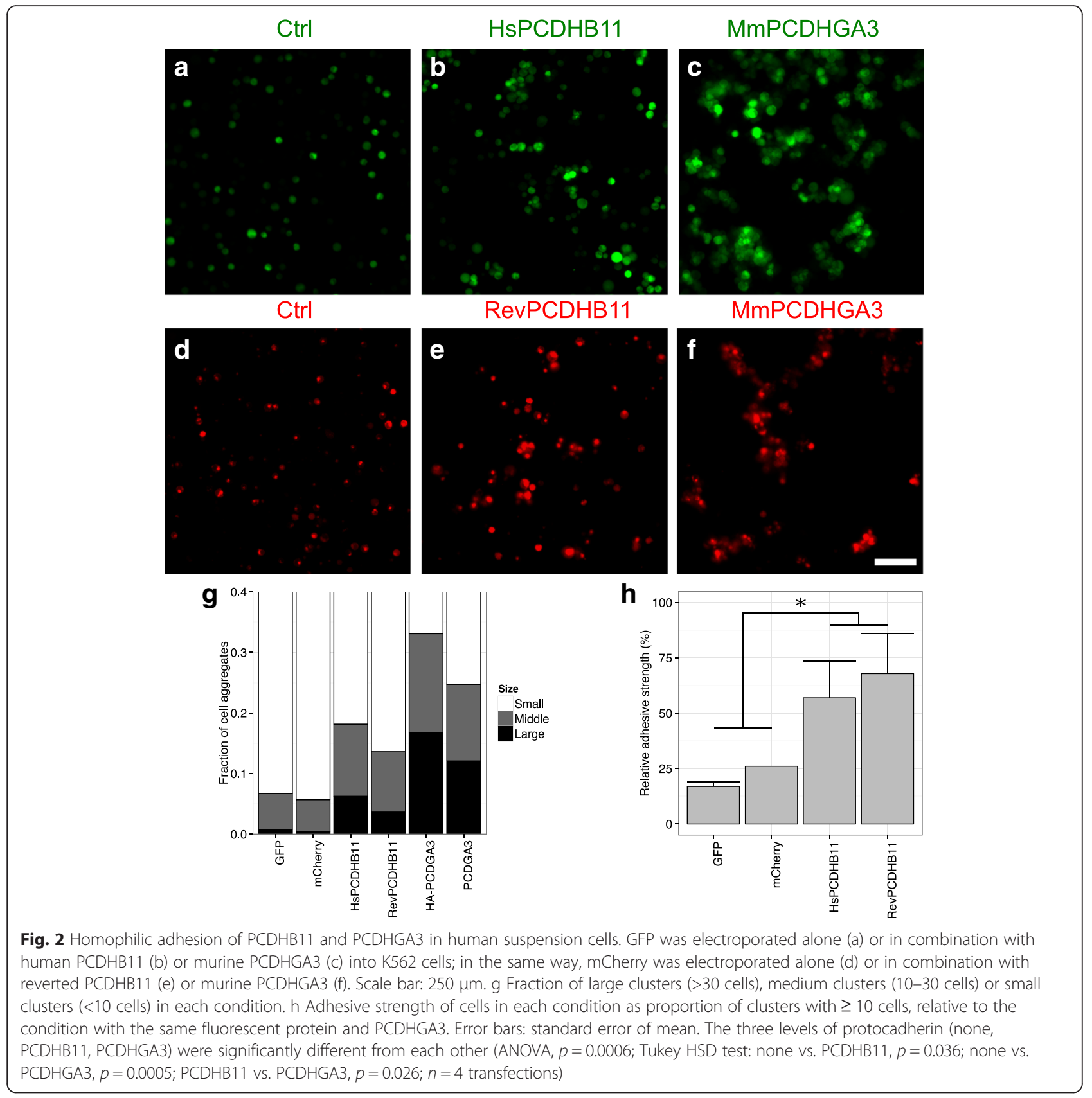

Evolutionary studies, such as the present one, might help to pinpoint molecules important for human functioning [54]. The most promising candidate from the bioinformatical part of the work was the $\beta$ protocadherin PCDHB11.

The $\beta$-protocadherin cluster, as a whole, was shown to be rapidly diverging between humans and chimpanzees [24]. According to the Allen Brain Atlas [55], PCDHB11 mRNA is expressed in the human brain, especially in the hippocampus, striatum, substantia nigra and locus ceruleus [56]. Human PCDHB11 has no direct murine homolog [57], but murine $\beta$ - protocadherin mRNAs are expressed combinatorially in Purkinje cells [58]. At the protein level, some murine $\beta$-protocadherins have been shown to be expressed in synapses of the central nervous system $[59,60]$. With regard to the physiological importance of $\beta$-protocadherins, the only data in humans so far have revealed a very high expression of PCDHB11 and PCDHB13 on the melanoma cell surface $[61,62]$. Hypermethylation of promoters in the protocadherin clusters, collectively, which is expected to downregulate gene expression, is a signal of Wilms' tumor [63], breast cancer [64] and neuroblastoma [65-67]. 

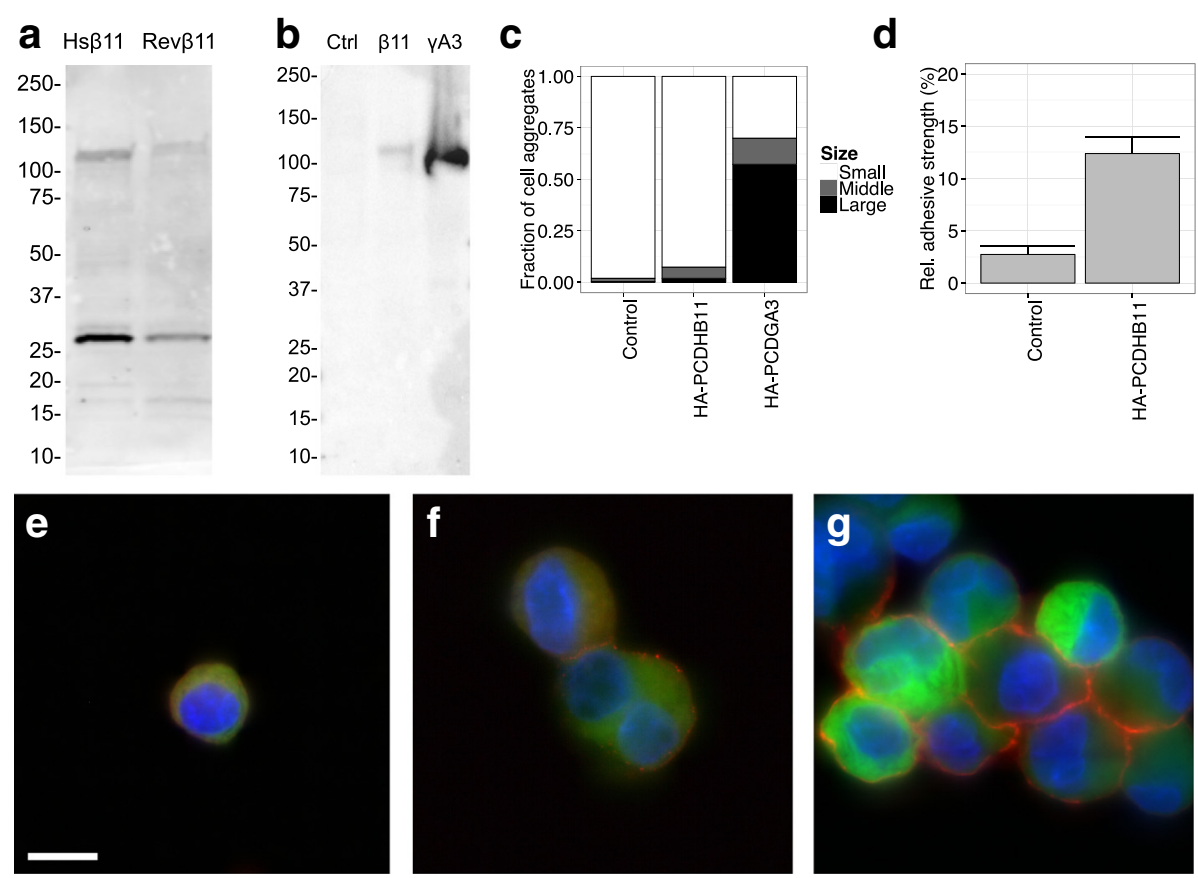

Fig. 3 Expression of protocadherins. GFP was electroporated alone or in combination with protocadherins into K562 cells. a Immunoblotting of cell lysates with anti-GFP shows bands compatible with the expected molecular weights for GFP (27 kDa) and mature PCDHB11-GFP (110 kDa); image representative of three transfections. $b$ Immunoblotting of cell lysates with anti-HA shows bands compatible with the expected molecular weights for mature HA-PCDHB11-GFP (113 kDa) and HA-PCDHGA3 (99 kDa); image representative of two transfections. c Proportion of medium and large clusters was lower in HA-PCDHB11-GFP-transfected than in HA-PCDHGA3-transfected cells, but higher than in control cells $(n=10-12$ images per condition). d Proportion of medium to large cell clusters when normalized by HA-PCDHGA3. For visualization of surface protocadherins, live cells transfected with GFP alone (e) or in combination with HA-PCDHB11-GFP (f) or HA-PCDHGA3 (g) were stained with antiHA-biotin and streptavidin-Alexa555, then fixed. Blue: DAPI. Green: GFP. Red: surface HA-tagged protocadherins. Scale bar: $10 \mu \mathrm{m}$

The most widely accepted model for the operation of clustered protocadherins is homophilic adhesion, where a hetero-oligomer of $\alpha$-, $\beta$ - and/or $\gamma$-protocadherins on the surface of one cell binds to an oligomer of the exact same composition on the surface of another cell [43, 48, 51]. While this homophilic binding can be measured as the formation of cell clusters in the K562 cell line model, in neurons it is proposed to lead to synapse disruption and therefore dendritic self-avoidance, optimizing the coverage of a neuron's territory [46, 51]. The present data extend this established cell culture model for the first time to a human protocadherin. Human PCDHB11 does induce the formation of $\mathrm{K} 562$ cell clusters, but at a very low level, and this low adhesivity has apparently been characteristic of PCDHB11 since before the divergence of human and chimpanzees. It is unclear if such a low level of adhesivity has functional relevance in the intact organism.

As homophilic adhesion is the main function of clustered protocadherins investigated experimentally so far, these results discourage a functional interpretation of the human-specific substitutions and require considering neutral evolution of this locus [68]. However, the unremarkable intra-human variability of $P C D H B 11$ suggests that there is no mutational hot spot at work, nor have selection constraints on $P C D H B 11$ been specially relaxed. No exon of $P C D H B 11$ was found to have high levels of biased gene conversion [69]. Moreover, while gene conversion events in certain human protocadherins have been reported, these events concentrated on the EC6 and cytoplasmic domains, sparing the EC1-EC3 domains [70]. Nine of twelve changes in PCDHB11 are located in EC2 and EC3, and it has been suggested, in a joint analysis of all human protocadherins, that positively selected positions are concentrated in these domains [71]. Finally, we note that the lack of increased intra-human variability also seems to discourage the hypothesis of diversifying selection.

While some other neutral process may yet explain the high density of human-specific substitutions in PCDHB11, it is also possible that selection might have occurred on an as yet unknown function of human PCDHB11. The subcellular localization of chick $\alpha-$ protocadherins [72] and rodent and primate $\beta$ protocadherins $[59,60]$ was suggested to be incompatible with the commonly assumed homophilic adhesion function. While no data on possible trans-interaction partners of $\beta$-protocadherins are available, heterophilic 

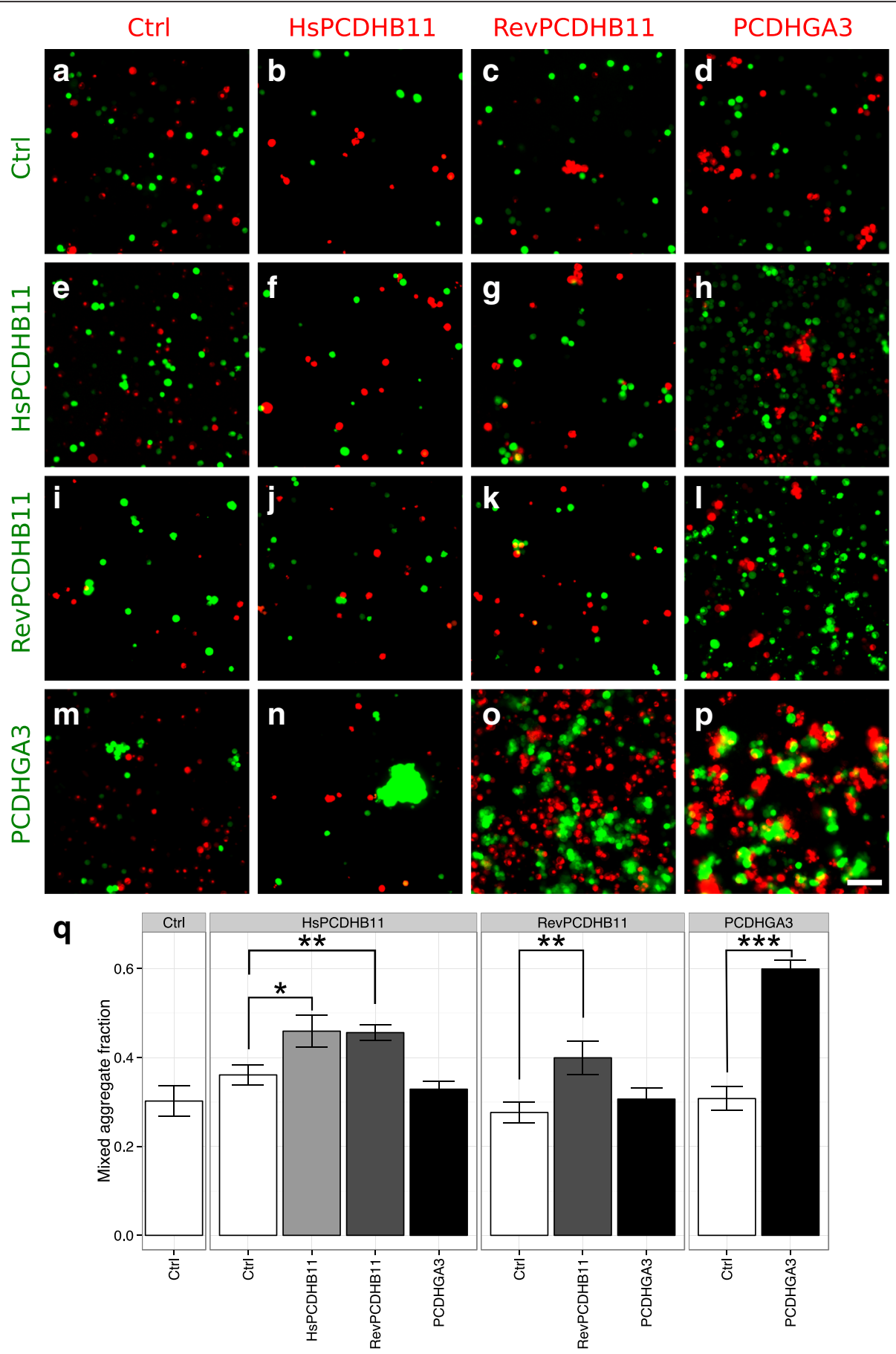

Fig. 4 Specificity of adhesion of PCDHB11. Different populations of K562 cells were separately electroporated as in Fig. 2, where one population received GFP and a protocadherin, and the other mCherry and a protocadherin. Green and red populations were mixed 24-48 $\mathrm{h}$ later, rocked for $2 \mathrm{~h}$ and then photographed while still alive. a-p The identity of the protocadherins in the green and red populations are indicated. Scale bar: $250 \mu \mathrm{m}$. q For each pair of cell populations, the conditions with switched fluorophores were joined, and the proportion of mixed clusters (containing both red and green cells) was expressed as fraction of all clusters. In an overall 2-way ANOVA, the identity of the cell populations and their interaction were each highly significant $\left(n=17-84\right.$ images per condition, $\left.p<10^{-8}\right)$. In each subpanel, the identity of the second cell population was significant (ANOVA, $p<0.05$ ). In each subpanel, the second cell populations were significantly different from control where indicated (linear models with Bonferroni correction for comparison with control; ${ }^{*} p<0,05 ;{ }^{* *}: p<0.01 ;{ }^{* * *}: p<0.001$ ) 


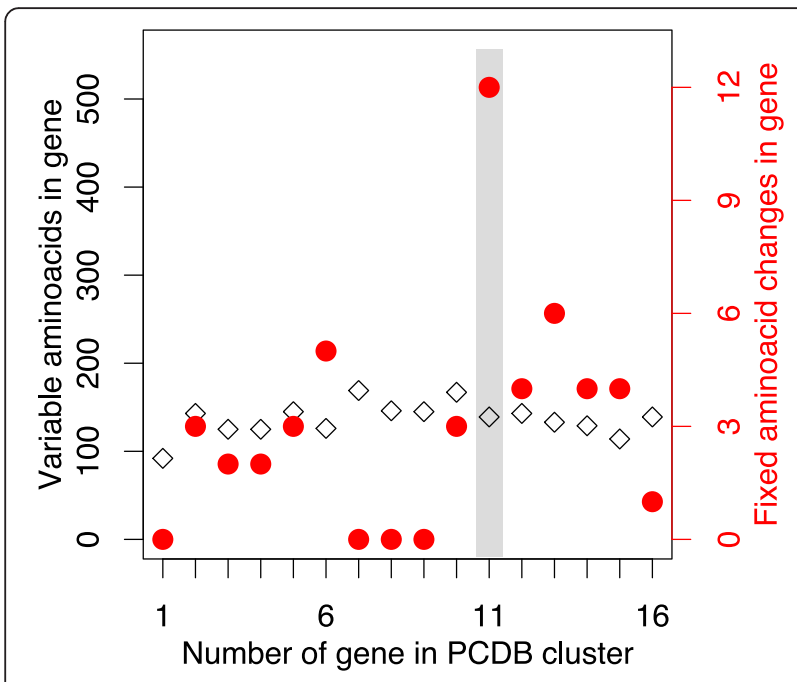

Fig. 5 Intra-human variability of PCDHB11 is not elevated. For each protein in the human $P C D H B$ cluster, the number of positions subject to intra-human variability is shown as black open diamonds (scale on left axis) and the number of substitutions on the human lineage as red full circles (scale on right axis, same data as in Table 1). Shaded grey bar: PCDHB11

adhesion to integrin has been proposed for murine $\alpha$ protocadherins [49], and additional intracellular roles distinct from cell adhesion have been suggested for some murine $\gamma$-protocadherins $[39,41,42]$.

\section{Conclusion}

The strategy employed here resulted in the discovery of PCDHB11 as a candidate gene for positive selection on the human lineage, combining a high number of potentially relevant substitutions on the human lineage with low intra-human variability. In spite of these genetic results, cell-based tests on the only proposed function for PCDHB11 did not indicate a functional change. If the molecular bases of human cognitive capacities can indeed be pinpointed to specific parts of the genome, they may well be discovered in changes affecting expression levels, such as copy number variations or non-coding regulatory sequences.

\section{Methods}

\section{Bioinformatics}

A list of genome positions where a panel of 100 human haploid genomes agrees on one base, while the reference genomes of common chimpanzee (Pan troglodytes), orangutan (Pongo abelii) and rhesus macaque (Macaca mulatta) agree on a different base, was kindly supplied by Martin Kircher [34]. For the existing sequence data, such a procedure was considered more prudent than classification of amino acid positions into different variability classes [73], both because of the small phylogenies and because structural information is not available for all proteins in the set. Human proteins where this base difference resulted in an amino acid difference were retained for further analysis if they were associated with the Gene Ontology terms [74]:

(1) "integral component of plasma membrane" or "anchored component of external side of plasma membrane", and additionally

(2)any term beginning with "nervous", "neuron", "dendr", "axon" or "synap" (except for those containing "enteric", "autonomous", "synaptonem", "axonem" or "dendritic cell”).

Since the surface annotation in this data base was incomplete, all members of a protein family (defined as those proteins having UNIPROT codes [75] beginning with the same three letters) were included if at least one member had passed the cell surface filter. The 329 resulting proteins were ranked by number of amino acid substitutions and also by number of substitutions normalized to the length of the protein.

The BLOSUM100 matrix gives the rate of one amino acid being exchanged for another one when calibrated for protein sequences of $>99 \%$ identity [76], corresponding to the overall identity between human and chimpanzee proteins [24]; the matrix was converted into weights, so that the most common exchanges had a weight of 3 and the least common exchange a weight of 15 . These weights were added up for all differences in each protein. Furthermore, the weighted number of differences was normalized by the length of the protein. To test the reliability of the ranking, the same procedure was repeated with the BLOSUM62 matrix (which is more commonly used) and with the JTT matrix (which was suggested by PROTTEST [73] as the best model for an evolutionary tree of PCDHB11), and changing the matrix did not strongly affect the ranking. All analyses were done using custom-written Python scripts.

For examination of surface exposure, human PCDHB11 and all protocadherins discussed in references [51, 52] were aligned using Clustal Omega [77]. The amino acids that correspond to human-specific PCDHB11 substitutions were localized in structure 4ZPL (murine PCDHB1, the nearest homolog that has been crystallized), 4ZI9 (murine PCDHGC3) and 4ZI8 (murine (PCDHGA1).

\section{Molecular biology}

Human PCDHB11 cDNA was ordered from imaGenes (Berlin, Germany), amplified by PCR using primers containing NheI and BamHI sites, and cloned into pEGFP-N1, so that GFP was added in frame at the C-terminus. HA-tagged PCDHB11-GFP was constructed by cloning annealed oligonucleotides coding for the HA-tag YPYDVPDYAE (Lifetech, São Paulo, 
Brasil) after the signal peptide cleavage site of PCDHB11. A reverted version was constructed by substituting the 12 consensus bases of the other primates into the human PCDHB11-GFP sequence, which was then ordered from Genscript (Piscataway, $\mathrm{NJ})$. The plasmids pcDNA3-HA-MmPCDGA3 and pmRFP-MmPCDGA3, coding for Mus musculus $\gamma$ protocadherin A3, were a kind gift from Dr. Dietmar Schreiner. All constructs were checked by Sanger sequencing of the entire open reading frame (Sequencing Core Facility, Institute of Biophysics, Federal University of Rio de Janeiro).

The number of variable positions in modern humans was calculated for each member of the $P C D H B$ cluster as the sum of missense, nonsense, stop lost and frame shift mutations in dbSNP [78].

\section{Cell culture}

K562 cells, an immortalized cell line derived from human leukemia [79], were a kind gift of Martin Bonamino, National Institute for Cancer Research, Rio de Janeiro, Brasil. K562 cells were cultured in a rich medium [80], and $2 \cdot 10^{6}$ cells were electroporated with $1 \mu \mathrm{g}$ of pEGFP-N1 or membrane-anchored mCherry plus $5 \mu \mathrm{g}$ of the indicated protocadherin plasmid DNA, using "1 M" buffer and Mirus Biotech (Madison, WI) or Bio-Rad (São Paulo, Brasil) cuvettes on a Nucleofector II (Lonza, Basel, Switzerland) [80]. Immediately after electroporation, cells were resuspended in $1 \mathrm{ml}$ of the culture medium and diluted to $8 \mathrm{ml}$ in warm culture medium. On the following day, where indicated, cells from different electroporation conditions were mixed 1:1 in 6-well plates. The plates were rocked for at least $2 \mathrm{~h}$ at $37{ }^{\circ} \mathrm{C}$ and 5-6 movements per minute, and 5-10 fields of view in each well were photographed on an Eclipse TE300 microscope using a 10x magnifying objective and a DS-QiMC camera (Nikon, Melville, NY).

For immunoblotting, transfected cells were centrifuged $10 \mathrm{~min}$ at $200 \mathrm{~g}, 37{ }^{\circ} \mathrm{C}$, and the supernatants were resuspended in radioimmunoprecipitation assay (RIPA) buffer including protease inhibitors (Thermo Fisher Scientific, Waltham, MA). $100 \mu \mathrm{g}$ (for GFP) or $20 \mu \mathrm{g}$ (for HA) of total protein were applied per well of a 6-15\% gradient Tris-Glycine gel (Bio-Rad Laboratories, Hercules, CA), and after eletrophoresis transferred to a nitrocellulose membrane (GE Healthcare, Little Chalfont, UK). For GFP detection, membranes were incubated with a rabbit polyclonal antibody (Life Technologies, A-11122) diluted 1:2000 in $50 \mathrm{mM}$ Tris, $100 \mathrm{mM} \mathrm{NaCl}, 0.1 \%$ Tween 20, $\mathrm{pH}$ 7.4, with $3 \%$ bovine serum albumine (Sigma, St. Louis, MO) and a horse-radish peroxidase-conjugated anti-rabbit secondary antibody (Invitrogen, Waltham, MA), while for HA revelation, they were incubated with biotinylated 3 F10 rat monoclonal antibody (Roche Life Science, Indianapolis, IN) diluted 1:500 in the same buffer, and streptavidin-horseradish peroxidase (Invitrogen, Waltham, MA). All membranes were revealed on a ChemiDoc system (Bio-Rad) using Super Signal West Femto (Thermo Fisher Scientific).

For HA staining, live K562 cultures were incubated for one hour with the biotinylated $3 \mathrm{~F} 10$ anti-HA, then for 40 min with streptavidin-Alexa555, both diluted 1:250 in culture medium at $8{ }^{\circ} \mathrm{C}$, then fixed for $15 \mathrm{~min}$ in $4 \%$ paraformaldehyde, $4 \%$ sucrose in phosphate-buffered saline at $8{ }^{\circ} \mathrm{C}$, deposited on slides using a CytoSpin (Thermo Fisher Scientific) and mounted in ProLong containing 4',6-diamidino-2-phenylindole (DAPI; Invitrogen). Images were taken on an Axiovert $200 \mathrm{M}$ microscope, using a 100x objective (Zeiss, Göttingen, Germany).

\section{Quantification}

For quantification of aggregate size, cell clusters were thresholded in ImageJ 2.0.0 [81] using the triangle algorithm implemented in the AutoThreshold plugin for ImageJ [82], and all areas of at least 100 pixels were exported for analysis in $R$ [83]. From the size of a single cell, defined as the mode cluster size of 150 pixels for control GFPtransfected cells, the cutoff for clusters of 10 cells was calculated as 696 pixels (assuming spherical clusters), and for clusters of 30 cells as 1448 pixels. For statistical analysis, the fraction of clusters containing at least 10 cells was calculated for each condition and day. After normalizing each condition on each day to the PCDHGA3 condition on the same day with the same fluorophore, there was no significant difference in fraction of medium and large clusters between human and reverted PCDHB11 $(n=4$ transfections). The two control conditions, the two PCDHB11 conditions and the two PCDHGA3 conditions were therefore pooled for further analysis.

For quantification of adhesion specificity, the separate green and red channels of each field were autothresholded as above, and the two masks were added. The presence of thresholded green and red cells was recorded for each cluster. In $\mathrm{R}$, the proportion of clusters containing both green and red cells, relative to all clusters, was calculated for each image.

\section{Ethics}

In vitro research using already derived and established human cell lines such as the immortalized K562 cells used in this publication, from which the identity of the donor(s) cannot readily be ascertained by the investigator, are not considered human subject research, and institutional review is not required for such research.

\section{Consent to publish}

Not applicable. 


\section{Availability of data and materials}

No human or animal samples were sequenced in this work. The dataset of human sequences supporting the conclusions of this article is freely available in the European Nucleotide Archive, accession number ERP000125 [84].

\section{Abbreviations}

DAPI: 4',6-diamidino-2-phenylindole; EC1-6: extracellular cadherin domain 16; GFP: green fluorescent protein; HA: hemagglutinin tag.

\section{Competing interests}

The authors declare that they have no competing interests.

\section{Authors' contributions}

MG conceived the study, performed bioinformatical analyses, participated in the experiments, performed statistical analyses and wrote the manuscript. GBF and RAG participated in the experiments. All authors have read and approved the final version of the manuscript.

\section{Acknowledgements}

The authors are grateful to Martin Kircher for sharing the evolutionary data base, to Svante Pääbo for support in the initial stage of the project; to Sérgio T. Ferreira and Fernanda G. De Felice for the generous permission to use their cell culture laboratory and other equipment; to Carla P. Ribeiro for use of the Nucleofector; to Martin Bonamino for the gift of the K562 cells; and to Dietmar Schreiner for the gift of the PCDGA3 plasmids. Carolina M. de Souza, Jessica F. S. Marques, Désirée O. S. de Figueiredo, Manuella Barata and Rafael M. Silva participated in earlier stages of this project.

\section{Funding}

This study was supported by grants from the Fundação Carlos Chagas Filho de Amparo à Pesquisa do Estado do Rio de Janeiro (FAPERJ; grant numbers E-26/111.541/2010, E-26/110.372/2012, E-26/111.517/2013) and Conselho Nacional de Desenvolvimento Científico e Tecnológico (CNPq; grant numbers 477130/2010-0 and 477959/2012-1) to MG. GBF received a fellowship from CNPq. RAG received fellowships from CNPq and FAPERJ. The funders had no role in study design, collection, analysis and interpretation of the data or writing the manuscript.

Received: 23 February 2016 Accepted: 6 April 2016

Published online: 12 April 2016

\section{References}

1. Herculano-Houzel S. The remarkable, yet not extraordinary, human brain as a scaled-up primate brain and its associated cost. Proc Natl Acad Sci U S A. 2012;109:10661-8.

2. Enard W, Gehre S, Hammerschmidt K, Hölter SM, Blass T, Somel M, et al. A humanized version of Foxp2 affects cortico-basal ganglia circuits in mice. Cell. 2009;137:961-71.

3. Reimers-Kipping S, Hevers W, Pääbo S, Enard W. Humanized Foxp2 specifically affects cortico-basal ganglia circuits. Neuroscience. 2011;175: 75-84.

4. Schreiweis C, Bornschein U, Burguière E, Kerimoglu C, Schreiter S. Humanized FOPX2 accelerates learning by enhancing transitions from declarative to procedural performance. Proc Natl Acad Sci U S A. 2014;111: 14253-8.

5. Hayakawa T, Angata T, Lewis AL, Mikkelsen TS, Varki NM, Varki A. A humanspecific gene in microglia. Science. 2005;309:1693.

6. Dennis MY, Nuttle X, Sudmant PH, Antonacci F, Graves TA, Nefedov M, et al. Evolution of human-specific neural SRGAP2 genes by incomplete segmental duplication. Cell. 2012;149:912-22.

7. Charrier C, Joshi K, Coutinho-Budd J, Kim JE, Lambert N, De Marchena J, et al. Inhibition of SRGAP2 function by its human-specific paralogs induces neoteny during spine maturation. Cell. 2012;149:923-35.

8. Florio M, Albert M, Taverna E, Namba T, Brandl H, Lewitus E, et al. Humanspecific gene ARHGAP11B promotes basal progenitor amplification and neocortex expansion. Science. 2015;347:1465-70.

9. King M, Wilson A. Evolution at two levels in humans and chimpanzees. Science. 1975;188:107-16.
10. Marnetto D, Molineris I, Grassi E, Provero P. Genome-wide identification and characterization of fixed human-specific regulatory regions. Am J Hum Genet. 2014;95:39-48.

11. Kamm GB, Pisciottano F, Kliger R, Franchini LF. The developmental brain gene NPAS3 contains the largest number of accelerated regulatory sequences in the human genome. Mol Biol Evol. 2013;30:1088-102.

12. Peters BD, Voineskos AN, Szeszko PR, Lett TA, DeRosse P, Guha S, et al. Brain white matter development is associated with a human-specific haplotype increasing the synthesis of long chain fatty acids. J Neurosci. 2014;34: 6367-76.

13. Shi L, Lin Q, Su B. Human-specific hypomethylation of CENPJ, a key brain size regulator. Mol Biol Evol. 2014;31:594-604.

14. Slattery M, Zhou T, Yang L, Machado ACD, Gordân R, Rohs R. Absence of a simple code : how transcription factors read the genome. Trends Biochem Sci. 2014;39:381-99.

15. Lalueza-Fox C, Römpler H, Caramelli D, Stäubert C, Catalano G, Hughes D, et al. A melanocortin 1 receptor allele suggests varying pigmentation among Neanderthals. Science. 2007;318:1453-5.

16. Gralle M, Pääbo S. A comprehensive functional analysis of ancestral human signal peptides. Mol Biol Evol. 2010;28:25-8.

17. Burbano HA, Green RE, Maricic T, Lalueza-Fox C, de la Rasilla M, Rosas A, et al. Analysis of human accelerated DNA regions using archaic hominin genomes. PLoS One. 2012;7:1-8.

18. Liu X, Somel M, Tang L, Yan Z, Jiang X, Guo S, et al. Extension of cortical synaptic development distinguishes humans from chimpanzees and macaques. Genome Res. 2012;22:611-22.

19. Castellano S, Parra G, Sánchez-Quinto FA, Racimo F, Kuhlwilm M, Kircher M, et al. Patterns of coding variation in the complete exomes of three Neandertals. Proc Natl Acad Sci U S A. 2014;111:6666-71.

20. Rodríguez-Vidal J, D’Errico F, Pacheco FG, Blasco R, Rosell J, Jennings RP, et al. A rock engraving made by Neanderthals in Gibraltar. Proc Natl Acad Sci U S A. 2014;111:13301-6.

21. Peresani M, Dallatorre S, Astuti P, Dal Colle M, Ziggiotti S, Peretto C. Symbolic or utilitarian? Juggling interpretations of Neanderthal behavior: new inferences from the study of engraved stone surfaces. J Anthr Sci. 2014;92:233-55.

22. Wobber V, Herrmann E, Hare B, Wrangham R, Tomasello M. Differences in the early cognitive development of children and great apes. Dev Psychobiol. 2014;56:547-73.

23. Olson MV, Varki A. Sequencing the chimpanzee genome: insights into human evolution and disease. Nat Rev Genet. 2003;4:20-8.

24. Chimpanzee Sequencing Consortium Analysis. Initial sequence of the chimpanzee genome and comparison with the human genome. Nature. 2005:437:69-87.

25. Nielsen R, Bustamante C, Clark AG, Glanowski S, Sackton TB, Hubisz MJ, et al. A scan for positively selected genes in the genomes of humans and chimpanzees. PLoS Biol. 2005;3:e170.

26. Rhesus Macaque Genome Sequencing and Analysis Consortium. Evolutionary and biomedical insights from the rhesus macaque genome. Science. 2007;316:222-34.

27. Scally A, Dutheil JY, Hillier LW, Jordan GE, Goodhead I, Herrero J, et al. Insights into hominid evolution from the gorilla genome sequence. Nature. 2012:483:169-75.

28. Nozawa M, Suzuki Y, Nei M. Reliabilities of identifying positive selection by the branch-site and the site-prediction methods. Proc Natl Acad Sci U S A. 2009;106:6700-5.

29. Gralle M, Ferreira ST. Structure and functions of the human amyloid precursor protein: the whole is more than the sum of its parts. Prog Neurobiol. 2007;82:11-32.

30. Giagtzoglou N, Ly CV, Bellen HJ. Cell adhesion, the backbone of the synapse: "vertebrate" and "invertebrate" perspectives. Cold Spring Harb Perspect Biol. 2009;1:a003079.

31. Shapiro L, Love J, Colman DR. Adhesion molecules in the nervous system: structural insights into function and diversity. Ann Rev Neurosci. 2007;30: 451-74

32. Valnegri P, Sala C, Passafaro M. Synaptic plasticity. Dynamics, development and disease. In: Kreutz MP, Sala C, editors. Adv Exp Med Biol. Wien: Springer; 2012. p. 433-50.

33. Rietveld CA, Esko T, Davies G, Pers TH, Turley P, Benyamin B, et al. Common genetic variants associated with cognitive performance identified using the proxy-phenotype method. Proc Natl Acad Sci U S A. 2015;112:13790-4. 
34. Burbano HA, Hodges E, Green RE, Briggs AW, Krause J, Meyer M, et al. Targeted investigation of the Neandertal genome by array-based sequence capture. Science. 2010;328:723-5.

35. Ashburner M, Ball CA, Blake JA, Botstein D, Butler $\mathrm{H}$, Cherry JM, et al. Gene ontology: tool for the unification of biology. The Gene Ontology Consortium. Nat Genet. 2000;25:25-9.

36. Shapiro L, Colman DR. Structural biology of cadherins in the nervous system. Curr Opin Neurobiol. 1998;8:593-9.

37. Yagi T. Molecular codes for neuronal individuality and cell assembly in the brain. Front Mol Neurosci. 2012;5:1-11.

38. Reiss K, Maretzky T, Haas IG, Schulte M, Ludwig A, Frank M, et al. Regulated ADAM10-dependent ectodomain shedding of gamma-protocadherin C3 modulates cell-cell adhesion. J Biol Chem. 2006;281:21735-44.

39. Fernández-Monreal M, Kang S, Phillips GR. Gamma-protocadherin homophilic interaction and intracellular trafficking is controlled by the cytoplasmic domain in neurons. Mol Cell Neurosci. 2009;40:344-53.

40. Garrett AM, Weiner JA. Control of CNS synapse development by gammaprotocadherin-mediated astrocyte-neuron contact. J Neurosci. 2009;29: 11723-31.

41. Fernández-Monreal M, Oung T, Hanson HH, O'Leary R, Janssen WG, Dolios $G$, et al. Gamma-protocadherins are enriched and transported in specialized vesicles associated with the secretory pathway in neurons. Eur J Neurosci. 2010;32:921-31.

42. Hanson HH, Kang S, Fernández-Monreal M, Oung T, Yildirim M, Lee R, et al. LC3-dependent intracellular membrane tubules induced by gammaprotocadherins A3 and B2: a role for intraluminal interactions. J Biol Chem. 2010;285:20982-92.

43. Schreiner $D$, Weiner JA. Combinatorial homophilic interaction between gamma-protocadherin multimers greatly expands the molecular diversity of cell adhesion. Proc Natl Acad Sci U S A. 2010;107:14893-8.

44. Chen WV, Alvarez FJ, Lefebvre JL, Friedman B, Nwakeze C, Geiman E, et al. Functional significance of isoform diversification in the protocadherin gamma gene cluster. Neuron. 2012;75:402-9.

45. Garrett AM, Schreiner D, Lobas MA, Weiner JA. y-Protocadherins control cortical dendrite arborization by regulating the activity of a FAK/PKC/ MARCKS signaling pathway. Neuron. 2012;74:269-76.

46. Lefebvre JL, Kostadinov D, Chen WV, Maniatis T, Sanes JR. Protocadherins mediate dendritic self-avoidance in the mammalian nervous system. Nature. 2012;488:517-21.

47. Li Y, Xiao H, Chiou T-T, Jin H, Bonhomme B, Miralles CP, et al. Molecular and functional interaction between protocadherin- $\gamma C 5$ and GABAA receptors. $J$ Neurosci. 2012;32:11780-97.

48. Thu CA, Chen W, Rubinstein R, Chevee M, Wolcott HN, Felsovalyi KO, et al. Single-cell identity generated by combinatorial homophilic interactions between $a, \beta$, and $\gamma$ protocadherins. Cell. 2014;158:1045-59.

49. Mutoh T, Hamada S, Senzaki K, Murata Y, Yagi T. Cadherin-related neuronal receptor 1 (CNR1) has cell adhesion activity with beta1 integrin mediated through the RGD site of CNR1. Exp Cell Res. 2004;294:494-508.

50. Katori S, Hamada S, Noguchi Y, Fukuda E, Yamamoto T, Yamamoto H, et al. Protocadherin-gamma family is required for serotonergic projections to appropriately innervate target brain areas. J Neurosci. 2009:29:9137-47.

51. Rubinstein R, Thu CA, Goodman KM, Wolcott HN, Bahna F, Mannepalli S, et al. Molecular logic of neuronal self-recognition through protocadherin domain interactions. Cell. 2015;163:629-42.

52. Nicoludis JM, Lau SY, Schärfe CPI, Marks DS, Weihofen WA, Gaudet R. Structure and sequence analyses of clustered protocadherins reveal antiparallel interactions that mediate homophilic apecificity. Structure. 2015;23:2087-98.

53. Miki R, Hattori $K$, Taguchi $Y$, Tada MN, Isosaka T, Hidaka $Y$, et al. Identification and characterization of coding single-nucleotide polymorphisms within human protocadherin-alpha and -beta gene clusters. Gene. 2005;349:1-14.

54. Vamathevan JJ, Hasan S, Emes RD, Amrine-Madsen H, Rajagopalan D, Topp $\mathrm{SD}$, et al. The role of positive selection in determining the molecular cause of species differences in disease. BMC Evol Biol. 2008;8:273.

55. Hawrylycz MJ, Lein ES, Guillozet-Bongaarts AL, Shen EH, Ng L, Miller JA, et al. An anatomically comprehensive atlas of the adult human brain transcriptome. Nature. 2012:489:391-9.

56. Human Brain Atlas. http://human.brain-map.org/microarray/search/ show? search term=PCDHB11. Accessed 22 Feb 2015

57. Vanhalst $K$, Kools $P$, Vanden Eynde E, van Roy F. The human and murine protocadherin-beta one-exon gene families show high evolutionary conservation, despite the difference in gene number. FEBS Lett. 2001;495:120-5.
58. Hirano K, Kaneko R, Izawa T, Kawaguchi M, Kitsukawa T, Yagi T. Singleneuron diversity generated by Protocadherin- $\beta$ cluster in mouse central and peripheral nervous systems. Front Mol Neurosci. 2012;5:1-13.

59. Junghans D, Heidenreich M, Hack I, Taylor V, Frotscher M, Kemler R. Postsynaptic and differential localization to neuronal subtypes of protocadherin beta16 in the mammalian central nervous system. Eur J Neurosci. 2008;27:559-71.

60. Puller C, Haverkamp S. Cell-type-specific localization of protocadherin $\beta 16$ at AMPA and AMPA/kainate receptor-containing synapses in the primate retina. J Comp Neurol. 2011;519:467-79.

61. Luo Y, Ellis LZ, Dallaglio K, Takeda M, Robinson WA, Robinson SE, et al. Side population cells from human melanoma tumors reveal diverse mechanisms for chemoresistance. J Invest Dermatol. 2012;132:2440-50.

62. Dobroff AS, Rodrigues EG, Juliano MA, Friaça DM, Nakayasu ES, Almeida IC, et al. Differential antitumor effects of lgG and lgM monoclonal antibodies and their synthetic regions directed to new targets of B16F10-Nex2. Transl Oncol. 2010;3:204-17.

63. Dallosso AR, Hancock AL, Szemes M, Moorwood K, Chilukamarri L, Tsai H-H, et al. Frequent long-range epigenetic silencing of protocadherin gene clusters on chromosome 5q31 in Wilms' tumor. PLoS Genet. 2009;5:e1000745.

64. Novak P, Jensen T, Oshiro MM, Watts GS, Kim CJ, Futscher BW. Agglomerative epigenetic aberrations are a common event in human breast cancer. Cancer Res. 2008;68:8616-25.

65. Abe M, Ohira M, Kaneda A, Yagi Y, Yamamoto S, Kitano Y, et al. CpG island methylator phenotype is a strong determinant of poor prognosis in neuroblastomas. Cancer Res. 2005;65:828-34.

66. Banelli B, Merlo DF, Allemanni G, Forlani A, Romani M. Clinical potentials of methylator phenotype in stage 4 high-risk neuroblastoma : an open challenge. PLoS One. 2013:8:63253.

67. Asada K, Abe M, Ushijima T. Clinical application of the CpG island methylator phenotype to prognostic diagnosis in neuroblastomas. J Hum Genet. 2013;1-6. doi: 10.1038/jhg.2013.64

68. Nei M, Fuerst PA, Chakraborty R. Testing the neutral mutation hypothesis by distribution of single locus heterozygosity. Nature. 1976;262:491-3.

69. Berglund J, Pollard KS, Webster MT. Hotspots of biased nucleotide substitutions in human genes. PLoS Biol. 2009;7:e26.

70. Noonan JP, Grimwood J, Schmutz J, Dickson M, Myers RM. Gene conversion and the evolution of protocadherin gene cluster diversity. Genome Res. 2004;14:354-66

71. Wu Q. Comparative genomics and diversifying selection of the clustered vertebrate protocadherin genes. Genetics. 2005;169:2179-88.

72. Blank M, Triana-Baltzer GB, Richards CS, Berg DK. Alpha-protocadherins are presynaptic and axonal in nicotinic pathways. Mol Cell Neurosci. 2004;26: 530-43.

73. Abascal F, Zardoya R, Posada D. ProtTest: Selection of best-fit models of protein evolution. Bioinformatics. 2005:21:2104-5.

74. Gene ontology database. ftp://ftp.ebi.ac.uk/pub/databases/GO/goa/HUMAN/ gene_association.goa_human.gz. Accessed 5 Jan 2010.

75. Uniprot database. http://www.uniprot.org/uniprot. Accessed 5 Jan 2010

76. Henikoff S, Henikoff JG. Performance evaluation of amino acid substitution matrices. Proteins. 1993;17:49-61.

77. Sievers F, Wilm A, Dineen D, Gibson TJ, Karplus K, Li W, et al. Fast, scalable generation of high-quality protein multiple sequence alignments using Clustal Omega. Mol Syst Biol. 2011;7:539.

78. dbSNP. http:/http://www.ncbi.n/m.nih.gov/variation/view/PCDHB11/. Accessed 20 Feb 2015.

79. Klein E, Ben-Bassat H, Neumann H, Ralph P, Zeuthen J, Polliack A, et al. Properties of the K562 cell line, derived from a patient with chronic myeloid leukemia. Int J Cancer. 1976:18:421-31.

80. Chicaybam L, Sodre AL, Curzio BA, Bonamino MH. An efficient low cost method for gene transfer to T Iymphocytes. PLoS One. 2013;8:e60298.

81. Schindelin J, Arganda-Carreras I, Frise E, Kaynig V, Longair M, Pietzsch T, et al. Fiji: an open-source platform for biological-image analysis. Nat Methods. 2012;9:676-82

82. Landini G. Advanced shape analysis with ImageJ. Proceedings of the Second ImageJ User and Developer Conference. Luxembourg; 2008. pp. 116-121.

83. R Core Team. R: A language and environment for statistical computing. Vienna: R Foundation for Statistical Computing; 2014.

84. European nucleotide archive. http://www.ebiacuk/ena/data/view/ ERP000125. Accessed 4 Apr 2016 